\title{
ACO with Intuitionistic Fuzzy Pheromone Updating Applied on Multiple-Constraint Knapsack Problem
}

\author{
Stefka Fidanova ${ }^{1, *}$ and Krassimir Todorov Atanassov ${ }^{2}$ \\ 1 Institute of Information and Communication Technologies, Bulgarian Academy of Sciences, Acad. G. \\ Bonchev Str. Bl. 25A, 1113 Sofia, Bulgaria \\ 2 Institute of Biophysics and Biomedical Engineering, Bulgarian Academy of Sciences, Acad. G. Bonchev Str. \\ Bl. 105, 1113 Sofia, Bulgaria; krat@bas.bg \\ * Correspondence: stefka.fidanova@iict.bas.bg
}

Citation: Fidanova, S.; Atanassov, K.T. ACO with Intuitionistic Fuzzy Pheromone Updating Applied on Multiple-Constraint Knapsack Problem . Mathematics 2021, 9, 1456. https://doi.org/10.3390/math9131456

Academic Editors: Basil

Papadopoulos and Michael Voskoglou

Received: 20 April 2021

Accepted: 19 June 2021

Published: 22 June 2021

Publisher's Note: MDPI stays neutral with regard to jurisdictional claims in published maps and institutional affiliations.

Copyright: (c) 2021 by the authors. Licensee MDPI, Basel, Switzerland. This article is an open access article distributed under the terms and conditions of the Creative Commons Attribution (CC BY) license (https:/ / creativecommons.org/licenses/by/ $4.0 /)$.

\begin{abstract}
Some of industrial and real life problems are difficult to be solved by traditional methods, because they need exponential number of calculations. As an example, we can mention decisionmaking problems. They can be defined as optimization problems. Ant Colony Optimization (ACO) is between the best methods, that solves combinatorial optimization problems. The method mimics behavior of the ants in the nature, when they look for a food. One of the algorithm parameters is called pheromone, and it is updated every iteration according quality of the achieved solutions. The intuitionistic fuzzy (propositional) logic was introduced as an extension of Zadeh's fuzzy logic. In it, each proposition is estimated by two values: degree of validity and degree of non-validity. In this paper, we propose two variants of intuitionistic fuzzy pheromone updating. We apply our ideas on Multiple-Constraint Knapsack Problem (MKP) and compare achieved results with traditional ACO.
\end{abstract}

Keywords: ant colony optimization; artificial intelligence; intuitionistic fuzzy pheromone; metaheuristics; multiple-constraint knapsack problem; nature inspired methods

\section{Introduction}

Difficult optimization problems arise very often in engineering applications. The complexity increases when the size of the solved problem increases, making it impossible to solve the problem with exact methods or traditional numerical methods. It is a great incentive to attract the attention of the scientists to develop new methods. The methods need to be flexible, easy for application, and to be applicable on various classes of problems, without significant changes.

Nature-inspired methods are in the group of artificial intelligent methods and are very convenient for solving NP-hard optimization problems. They are based on ideas coming from some natural phenomena: biological, physical, chemical, etc. These methods can be divided to several categories: evolutionary; swarm intelligence-based; physicsbased. Other categorization is: population-based; single solution-based. Representative of evolutionary-based algorithm is Genetic algorithm [1,2]. It simulates the Darwinian concept for evolution. Some physical concepts underlie algorithms based on physical phenomena. As representatives of this kind of algorithms, we will mention Simulated Annealing [3] and Gravitation search algorithm [4]. Interior search [5] and Tabu search [6] can be seen as a representative of human behavior algorithms. The behavior of animals and insects in the nature inspire algorithms based on swarm intelligence. Ant Colony Optimization [7], Artificial Bee Colony [8], Bat algorithm [9], Firefly algorithm [10], Particle Swarm Optimization [11], Gray Wolf algorithm [12], and Emperor Penguins Colony [13] are representative of the swarm intelligence of the animals, etc.

MKP is NP-hard combinatorial optimization problem and between most appropriate algorithms to solve it is Ant Colony Optimization (ACO). Observing ants, researchers have found that they can find the shortest path to a food source and back, even when 
there are obstacles. Marco Dorigo was the first scientist to propose a methodology based on the behavior of ants to solve complex optimization problems [14]. The solution is presented as a path in a graph that describes the problem. The best solution is the shorter path in a graph, which respects the problem constraints. Every ant construct solution applying probabilistic rule, starting from random node The real ants deposit on the ground a mucous substance called pheromone. Next, ants follow the path with most concentrated pheromone. Imitating real ants, in the algorithm a numerical value is related with the elements of the graph, similar to the pheromone in the nature. Thus, the elements of better solutions are marked with more pheromone and are used more subsequently. The pheromone intensifies the search in the regions with good solutions and random starts diversify the search inside the set of possible solutions. The combination of intensification and diversification helps to avoid local optimums.

Various types of combinatorial optimization problems can be solved with the help of ACO methodology. It is very suitable to be applied to problems with strict constraints, because it is a constructive method and does not require additional efforts to meet them. Thus, it outperforms other metaheuristic methods, in many cases.It gives a possibility for adaptation to dynamic changes of the problem [15-17]. The first application is on Traveling Salesman Problem (TSP). Over the years, the method has been successfully applied to a large number of combinatorial optimization problems. Several variants of ACO approaches exist: ant system [18]; elitist ants [18]; ant colony system [14]; max-min ant system [17]; rank-based ant system [18]; ant algorithm with additional reinforcement [19]. The pheromone updating is the main difference between the variants.

In this paper, we propose intuitionistic fuzzy variant of the pheromone updating. We test our variant of ACO algorithm on Multiple-Constraint Knapsack Problem (MKP). The MKP was chosen because it can be used to describe a wide variety of application problems arising in industry and real life.

The paper is organized in the following way: in Section 2, a short remark on intuitionistic fuzzy logic is given; the mathematical description of MKP is in Section 3; ACO algorithm with intuitionistic fuzzy pheromone updating is presented in Section 4; Section 5 is devoted to numerical experiments and their analysis; in Section 6, conclusion remarks are given.

\section{Short Remark on Intuitionistic Fuzzy Logic}

Intuitionistic fuzzy propositional logic is proposed by Krassimir Atanassov [20]. In the classical logic, to every proposition, it can be assigned value 1 or $0: 1$ in the sense of true and 0 in the sense of false. In fuzzy logic [21], there is a truth degree $m \in[0,1]$. In intuitionistic fuzzy (propositional) logic [22,23], each proposition is evaluated by two degrees: degree of validity (or of truth) $m \in[0,1]$ and degree of non-validity (or of false) $n \in[0,1]$, so that $m+n \leq 1$. These degrees generate a third degree $1-m-n$, called degree of uncertainty (or indeterminacy) and, obvious, the sum of all three components is 1 . The pair $\langle m, n\rangle$ is called an Intuitionistic Fuzzy Pair (IFP). For two IFPs a lot of operations, relations and operators are defined (see Reference [23]). For example, the simplest modal operators, defined over IFPs are: $\square\langle m, n\rangle=\langle m, 1-m\rangle, \diamond\langle m, n\rangle=\langle 1-n, n\rangle$.

\section{Multiple-Constraint Knapsack Problem}

Knapsack Problem is one of the most studied combinatorial optimization problem. Its name comes from the continuity, where is given a set of items with fixed volumes and values. The aim is to maximize the value of hall knapsack, without to exceeding the volume of the knapsack.

MKP is a generalization of single knapsack problem, where there are many knapsacks with diverse capacity. Each item is assigned to a maximum one knapsack. The aim is to maximize the total value of the knapsacks.

A lot of real problems can be represented by MKP. In Reference [24], they solve maritime shipping applications represented as MKP. The problem comes from Port of Shanghai, 
where a connection is made between river inland ports and maritime transportation network. The authors apply mixed-integer programming model to assign barges to tugs. Another application of MKP is considered in Reference [25]. They treat a problem in emergency situation when people at different locations are moved to safe place using a set of vehicles (knapsacks). It is shown that the problem is polynomial if the vehicles are identical, but when they are heterogeneous, the problem is solved by heuristic. In Reference [26], they solved a problem coming from the steel industry. Production planning demand assigning them to a set of production units. They consider the case when the profit and the weight of each item is the same. A variant of the problem with different weights and profit is studied in Reference [27]. They consider assignment to a base station of cell phone users. With a help of MKP can be modeled production planing with machine failures [28,29]. In Reference [30], algorithmic approaches to the MKP are discussed. MKP is a special case of the generalized assignment problem [31], and it is a representative of subset problems. Resource allocation in distributed systems, capital budgeting, cargo loading, and cutting stock problems can be defined as MKP [32]. Patient scheduling is an important real life problem. The patients are scheduled according their therapies, and the knapsacks correspond to the time and the therapies correspond to the items [33]. Bin packing problem [34] and cutting stock problem for cut row materials [32] are related with MKP, too. Another useful application is multi-processor scheduling [35]. The purpose is assignment of jobs with fixed processing times to the machines, minimizing the makespan. Another important applications of MKP are generation of keys in cryptosystems [32] and tests generations [36]. MKP models large set of binary problems with integer coefficients [36].

It is proved that MKP is NP-hard problem [37]. These kinds of problems are usually solved with metaheuristics, such as genetic algorithm [38], tabu search [39], and ant colony optimization [40]. Other swarm intelligence algorithms are applied in Reference [38,41]. Exact methods are applicable only for small size problems [37,42].

MKP will be defined as resource allocation problem in this paper, and $m$ is the number of resources (number of knapsacks), while $n$ is the number of objects. The profit of the object $j$ is $p_{j}$. The capacity $c_{i}$ of knapsack $i$ corresponds to the budget of the recourse $i$, and $r_{i j}$ is the consumption of recourse $i$ by object $j$. The objective is to maximize the profit in the framework of the limited budget. Mathematically, MKP can be described in the following way:

$$
\begin{gathered}
\max \sum_{j=1}^{n} p_{j} x_{j} \\
\text { subject to } \sum_{j=1}^{n} r_{i j} x_{j} \leq c_{i} i=1, \ldots, m . \\
x_{j} \in\{0,1\} \quad j=1, \ldots, n
\end{gathered}
$$

Let $I=\{1, \ldots, m\}$ and $J=\{1, \ldots, n\}$ and $c_{i} \geq 0$ for all $i \in I$. A well-stated MKP assumes that $p_{j}>0$ and $r_{i j} \leq c_{i} \leq \sum_{j=1}^{n} r_{i j}$ for all $i \in I$ and $j \in J$. The $\left[r_{i j}\right]_{m \times n}$ matrix and $\left[c_{i}\right]_{m}$ vector are both non-negative. The MKP solution is a binary string $\left\{i_{1}, i_{2}, \ldots, i_{n}\right\}$, the element $i_{j}$ for $j \in J$ is 1 if the $j$ th object is included in the knapsacks; otherwise, it is 0 .

\section{ACO Algorithm with Intuitionistic Fuzzy Pheromone}

As we mentioned above, exact methods and traditional numerical methods are applied only on small size NP-hard problems. The only possibility for large problems is the use of metaheuristics [18]. The metaheuristic methods find proximate solutions for reasonable computational resources. We apply ACO because it is one of the best metaheuristic methods. An ant marks its way with a substance called pheromone and is guided only by the concentration of the pheromone. To mimic the behavior of ants, the problem is represented by a graph, so the solutions can be represented by paths in a graph. In the case of MKP, we look for a longest path. The ants start their paths construction by a random node of the graph. A new node is included applying probabilistic rule, called transition 
probability. Transition probability $P_{l j}$ to select node $j$ after the node $l$ is a product of quantity of the pheromone $\tau_{l j}$ and heuristic information $\eta_{j},(l, j \in J)$.

$$
P_{l j}=\frac{\tau_{l j}^{a} \eta_{j}^{b}}{\sum_{k \in \text { Unused }} \tau_{l k}^{a} \eta_{k}^{b}},
$$

where Unused is a set of not used, by the ants, nodes.

The heuristic information represents the knowledge about the problem and is suitable combination of problem parameters. Let

$$
s_{j}=\sum_{i=1}^{m} r_{i j}
$$

and our heuristic information is:

$$
\eta_{j}=\left\{\begin{array}{cll}
p_{j} / s_{j} & \text { if } & s_{j} \neq 0 \\
p_{j} & \text { if } & s_{j}=0
\end{array} .\right.
$$

Thus, the objects with greater profit and less expense become more desirable. The aim of this heuristic is to include most profitable items and more items, which will lead to maximization of objective function.

The initial pheromone has a small positive value $\tau_{0}, \tau_{0} \in(0,1)$. When ants crate their solutions, the pheromone is updated. Let $\rho$ be the evaporation. The pheromone updating rule is:

$$
\tau_{l j} \leftarrow(1-\rho) \tau_{l j}+\Delta \tau_{l j}
$$

In most of the variants of ACO, $\Delta \tau_{l j}=\rho F$, where $F$ is the fitness function. Several variants of the methodology are proposed later. They affect mainly the pheromone updating $[18,19]$.

In this paper, we suggest using an intuitionistic fuzzy pheromone. Thus, the pheromone updating formula becomes:

$$
\tau_{l j} \leftarrow(1-\rho) \tau_{l j}+\alpha F,
$$

where $(1-\rho)+\alpha \leq 1, \alpha \in(0,1)$. In traditional ACO algorithms, the evaporation parameter $\rho$ is input data and stays unchanged during the run of the algorithm. In our case, we generate the parameter $\rho$ as a random number from the interval $(0,1)$; after that, the parameter $\alpha$ is generated as a random number in the interval $(0, \rho)$. Thus, it is guaranteed that the sum of the two parameters is less than 1 and the pheromone updating is intuitionistic fuzzy.

We propose two variants of intuitionistic fuzzy pheromone updating. In the first variant, the parameters $\rho$ and $\alpha$ are generated before the first iteration of the algorithm and stay unchanged during the hall iterations. Thus, in the same run, the values of the two parameters are the same, but, in a different runs of the algorithm, they are different. In the second variant, the parameters $\rho$ and $\alpha$ are generated at the beginning of each iteration. Thus, their values are different at each iteration during the same run. The aim of intuitionistic fuzzy pheromone updating is more diversification in a search process, which will give a possibility for finding better solutions.

\section{Experimental Results and Discussion}

The proposed two variants of intuitionistic fuzzy pheromone updating is tested on 10 MKP instances from Operational Research Library "OR-Library" available within WWW, accessed at http:/ /http:/ / people.brunel.ac.uk/ mastjjb/jeb / orlib / mknapinfo.html (21 Jun 2021). Every test problem consists of 100 items and 10 knapsacks/constraints. We prepare software, written in C programming language, which realizes the two variants of intuitionistic fuzzy pheromone updating. The tests are run on Pentium desktop computer at $2.8 \mathrm{GHz}$ with $4 \mathrm{~GB}$ of memory. We fixed the $\mathrm{ACO}$ parameters to the same for all tests and variants of the algorithm, as in Table 1 . The parameters are fixed experimentally. We run 
the algorithm several times with every one of the tests and varying the parameters, till we fixed their values. Thirty independent runs are performed with every of the test problems. Applying intuitionistic fuzzy pheromone updating, we aim larger diversification in a search. ANOVA test is applied, and it gives significance $95 \%$. It guarantee the difference between the average results.

Table 1. ACO parameter settings.

\begin{tabular}{cc}
\hline Parameters & Value \\
\hline Number of iterations & 300 \\
Number of ants & 20 \\
$\rho$ & 0.5 \\
$\tau_{0}$ & 0.5 \\
$a$ & 1 \\
$b$ & 1 \\
\hline
\end{tabular}

We want to note that both variants of the intuitionistic fuzzy pheromone updating do not affect the computational time. In Table 2, we report average results over 10 test problems, and every test is run 30 times. The second column reports the results with traditional ACO pheromone updating. The third column is ACO with intuitionistic fuzzy pheromone updating, when the parameters are calculated at the beginning of the algorithm. The forth column is ACO with intuitionistic fuzzy pheromone updating, when the parameters are calculated at the beginning of every iteration. The last row shows the average over every column. We observe that the intuitionistic fuzzy pheromone updating, for both variants, improves the achieved results in 8 of 10 tests, and the average over all 10 tests is better, too. Comparing two variants of intuitionistic fuzzy pheromone updating, we observe that the variant with calculation of the pheromone updating parameters at the beginning of the algorithm performs better, than the variant where the parameters are recalculated every iteration. The first variant achieves better results in 6 of 10 tests and the average over the all tests is better, too. We can conclude that applying intuitionistic fuzzy pheromone updating increases the diversification of the search and the chance of solution improvement. This diversification is more balanced when the pheromone updating parameters are calculated once, at the beginning of the algorithm. Thus, we can explain better algorithm performance in this case.

Table 2. ACO performance with different pheromone updating.

\begin{tabular}{cccc}
\hline Instance & Traditional ACO & Int Fuzzy Beginning & Int. Fuzzy Every \\
\hline MKP $100 \times 10^{-1}$ & 21989.43 & $\mathbf{2 2 0 6 1 . 0 0}$ & 21992.92 \\
MKP $100 \times 10^{-2}$ & 22059.90 & 22055.76 & $\mathbf{2 2 0 9 6 . 0 0}$ \\
MKP $100 \times 10^{-3}$ & 20989.00 & $\mathbf{2 1 0 2 0 . 5 6}$ & 20997.00 \\
MKP 100 $\times 10^{-4}$ & 21625.56 & 21656.03 & $\mathbf{2 1 6 6 6 . 6 6}$ \\
MKP 100 $\times 10^{-5}$ & 21718.33 & 21754.00 & $\mathbf{2 1 7 6 1 . 6 3}$ \\
MKP 100 $\times 10^{-6}$ & 21869.73 & $\mathbf{2 1 8 9 1 . 2 0}$ & 21867.16 \\
MKP 100 $\times 10^{-7}$ & 21477.30 & $\mathbf{2 1 5 0 9 . 5 3}$ & 21502.2 \\
MKP 100 $\times 10^{-8}$ & 21573.03 & $\mathbf{2 1 6 6 8 . 0 6}$ & 21596.16 \\
MKP 100 $\times 10^{-9}$ & $\mathbf{2 2 2 4 8 . 4 3}$ & 22229.46 & 2221.00 \\
MKP 100 $\times 10^{-10}$ & $\mathbf{4 0 5 8 1 . 5 1}$ & 40556.50 & 40578.10 \\
average & 23613.22 & $\mathbf{2 3 6 4 0 . 2 3}$ & 23627.88 \\
\hline
\end{tabular}

We prepare variant of ACO algorithm when parameter $\rho$ is fixed and only the parameter $\alpha$ is randomly generated, subject to the condition $(1-\rho)+\alpha \leq 1$, so keeping the intuitionistic fuzziness. The achieved results are statistically similar to the variant where both parameters, $\rho$ and $\alpha$, are randomly selected. Thus, we do not need tuning of the parameter $\rho$. 


\title{
6. Conclusions
}

In this paper, we propose ACO algorithm with two variants of intuitionistic fuzzy pheromone updating, where the sum of the two pheromone updating coefficients are less or equal to 1 . In the first variant, the parameters are generated before the first iteration and stay unchanged till the end of the algorithm. In the second variant, the parameters are generated at the beginning of every iteration. Our ideas are tested on $10 \mathrm{MKP}$ problems. Intuitionistic fuzzy pheromone updating is not time consuming. Both variants improve algorithm performance, comparing with traditional ACO. This can be explained by the greater diversification in the search. The variant with calculation of pheromone updating parameters at the beginning of the algorithm is more balanced and performs better than the variant with calculation of these parameters every iteration.

In the future, we will study other variants of the proposed ACO algorithm with use of the modal operators mentioned in Section 2 and of some of their extensions, described in Reference [22,23]. So, we will obtain optimistic, average, or pessimistic evaluation of the results of the algorithms.

Author Contributions: The authors contributed equally in this research. All authors have read and agreed to the published version of the manuscript.

Funding: The presented work is partially supported by the grant No BG05M2OP011-1.001-0003, financed by the Science and Education for Smart Growth Operational Program and co-financed by European Union through the European structural and Investment funds. The work is supported, too, by National Scientific Fund of Bulgaria under the grant DFNI DN 12/5.

Institutional Review Board Statement: Not applicable.

Informed Consent Statement: Not applicable.

Conflicts of Interest: The authors declare no conflict of interest.

\author{
Abbreviations \\ MDPI Multidisciplinary Digital Publishing Institute \\ DOAJ Directory of open access journals \\ TLA Three letter acronym \\ LD Linear dichroism
}

\section{References}

1. Goldberg, D.E.; Korb, B.; Deb, K. Messi Genetic Algorithms: Motivation Analysis and First Results. Complex Syst. 1989, 5, 493-530.

2. Yang, X.S. Nature-Inspired Metaheuristic Algorithms; Luniver Press: Beckington, UK, 2008.

3. Kirkpatrick, S.; Gelatt, C.D.; Vecchi, M.P. Optimization by simulated annealing. Science 1983, 13, 671-680. [CrossRef]

4. Mosavi, M.R.; Khishe, M.; Parvizi, G.R.; Naseri, M.J.; Ayat, M. Training multi-layer perceptron utilizing adaptive best-mass gravitational search algorithm to classify sonar dataset. Arch. Acoust. 2019, 44, 137-151.

5. Ravakhah, S.; Khishe, M.; Aghababaee, M.; Hashemzadeh, E. Sonar false alarm rate suppression using classification methods based on interior search algorithm. Comput. Sci. Netw. Secur. 2017, 17, 58-65.

6. Osman, I.H. Metastrategy simulated annealing and tabu search algorithms for the vehicle routing problem. Ann. Oper. Res. 1993, 41, 421-451. [CrossRef]

7. Bonabeau, E.; Dorigo, M.; Theraulaz, G. Swarm Intelligence: From Natural to Artificial Systems; Oxford University Press: Oxford, UK, 1999.

8. Karaboga, D.; Basturk, B. Artificial bee colony (ABC) optimization algorithm for solving constrained optimization problems. In Advances in Soft Computing: Foundations of Fuzzy Logic and Soft Computing; Springer: Haidelberg, Germany, 2007 ; pp. 789-798.

9. Vikhar, P.A. Evolutionary algorithms: A critical review and its future prospects. In Proceedings of the 2016 International Conference on Global Trends in Signal Processing, Information Computing and Communication (ICGTSPICC), Jalgaon, India, 22-24 December 2016; pp. 261-265.

10. Yang, X.S. A New Metaheuristic Bat-Inspired Algorithm. In Nature Inspired Cooperative Strategies for Optimization; Studies in Computational Intelligence, 284; Springer: Haidelberg, Germany, 2010; pp. 65-74.

11. Kennedy, J.; Eberhart, R. Particle Swarm Optimization. In Proceedings of the IEEE International Conference on Neural Networks IV, Perth, Australia, 27 November-1 December 1995; pp. 1942-1948.

12. Mirjalili, S.; Mirjalili, S.M.; Lewis, A. Grey Wolf Optimizer. Adv. Eng. Softw. 2014, 69, 46-61. [CrossRef] 
13. Harifi, S.; Khalilian, M.; Mohammadzadeh, J. Emperor Penguins Colony: A new metaheuristic algorithm for optimization. Evol. Intell. 2019, 12, 211-226. [CrossRef]

14. Dorigo, M.; Gambardella, L. Ant colony system: A cooperative learning approach to the traveling salesman problem. IEEE Trans. Evol. Comput. 1996, 1, 53-66. [CrossRef]

15. Fidanova, S.; Lirkov, I. 3d protein structure prediction. Analele Univ. Vest Timis. 2009, XLVII, 33-46.

16. Fidanova, S. An improvement of the grid-based hydrophobic-hydrophilic model. Bioautomation 2010, 14, 147-156.

17. Stutzle, T.; Hoos, H. Max min ant system. Future Gener. Comput. Syst. 2000, 16, 889-914. [CrossRef]

18. Dorigo M.; Stutzle T. Ant Colony Optimization; MIT Press: Cambridge, MA, USA, 2004.

19. Fidanova, S. ACO algorithm with additional reinforcement. In Proceedings of the From Ant Colonies to Artificial Ants Conference, Brussels, Belgium, 12-14 September 2003; pp. 292-293.

20. Atanassov, K. Two Variants of Intuitionistic Fuzzy Propositional Calculus. Bioautomation 2016, 20, 17-26.

21. Zadeh, L. Fuzzy Sets. Inf. Control. 1968, 12, 94-102. [CrossRef]

22. Atanassov, K. Intuitionistic Fuzzy Sets; Studies in Fuzziness and Soft Computing, 123; Springer: Heidelberg, Germany, 1999.

23. Atanassov, K. Intuitionistic Fuzzy Logics; Studies in Fuzziness and Soft Computing, 351; Springer: Heidelberg, Germany, 2017.

24. Zhen, L.; Wang, K.; Wang, S.; Qu, X. Tug scheduling for hinterland barge transport: a branch-and-price approach. Eur. J. Oper. Res. 2018, 265, 119-132. [CrossRef]

25. Dimitrov, N.B.; Solow, D.; Szmerekovsky, J.; Guo, J. Emergency relocation of items using single trips: special cases of the multiple knapsack assignment problem. Eur. J. Oper. Res. 2017, 258, 938-942. [CrossRef]

26. Dawande, M.; Kalagnanam, J.; Keskinocak, P.; Salman, F.S.; Ravi, R. Approximation algorithms for the multiple knapsack problem with assignment restrictions. Comb. Optim. 2000, 4, 171-186. [CrossRef]

27. Dahl, G.; Foldnes, N. LP based heuristics for the multiple knapsack problem with assignment restrictions. Ann. Oper. Res. 2006, 146, 91-104. [CrossRef]

28. Diaz, J.E.; Handl, J.; Xu, D.L. Integrating meta-heuristics, simulation and exact techniques for production planning of a failureprone manufacturing system. Eur. J. Oper. Res. 2018, 266, 976-989. [CrossRef]

29. Laalaoui, Y.; M'Hallah, R. A binary multiple knapsack model for single machine scheduling with machine unavailability. Comput. Oper. Res. 2016, 72, 71-82. [CrossRef]

30. Martello, S.; Monaci, M. Algorithmic approaches to the multiple knapsack assignment problem. Omega 2020, $90,102004$. [CrossRef]

31. Leguizamon, G.; Michalevich, Z. A new version of ant system for subset problems. In Proceedings of the Evolutionary Computations Conference, Washington, DC, USA, 6-9 July 1999; pp. 1459-1464.

32. Kellerer H.; Pferschy U.; Pisinger, D. Multiple Knapsack Problems. In Knapsack Problems; Springer: Heidelberg, Germany, 2004.

33. Arsik, I.; Keskinocak, P.; Coppola, J.; Hampapur, K.; He, Y.; Jiang, H.; Regala, D.; Tailhardat, N.; Goin, K. Effective and equitable appointment scheduling rehabilitation centers. In Proceedings of the INFORMS Annual Meeting, Huston, TX, USA, 22-25 October 2017.

34. Murgolo, F.D. An efficient approximation scheme for variable-sized bin packing. SIAM J. Comput. 2017, 16, 149-161. [CrossRef]

35. Lawler, E.L.; Lenstra, J.K.; Rinnooy Kan, A.H.G.; Shmoys, D.B. Sequencing and scheduling: algorithms and complexity. In Handbooks in OR and MS, 4; Graves, S.C., Kan, R., Zipkin, P.H., Eds.; Elsevier Science Publishers: Amsterdam, The Netherlands, 1993; pp. 445-522.

36. Feuerman, M.; Weiss, H. A Mathematical Programming Model for Test Construction and Scoring. Manag. Sci. 1973, 19, 961-966. [CrossRef]

37. Martello, S.; Toth, P. A heuristic algorithm for the multiple knapsack problem. Computing 1981, 27, 93-112. [CrossRef]

38. Liu Y.Y.; Wang S. A scalable parallel genetic algorithm for the generalized assignment problem. Parallel Comput. 2015, 46, 98-119. [CrossRef]

39. Woodcock A.J.; Wilson J.M. A hybrid tabu search/branch \& bound approach to solving the generalized assignment problem. Eur. J. Oper. Res. 2010, 207, 566-578.

40. Fidanova S.; Atanassov K. Flying Ant Colony Optimization Algorithm for Combinatorial Optimization. Studia Inform. 2017, $38,31-40$.

41. Krause, J.; Cordeiro, J.; Parpinelli, R.S.; Lopes, H.S. A survey of swarm algorithms applied to discrete optimization problems. In Swarm Intelligence and Bio-Inspired Computation; Elsevier Science Publishers: Amsterdam, The Netherlands, 2013 ; pp. 169-191.

42. Posta, M.; Ferland, J.A.; Michelon, P. An exact method with variable fixingfor solving the generalized assignment problem. Comput. Optim. Appl. 2012, 52, 629-644. [CrossRef] 